\title{
Health-Related Quality of Life and Functional Outcomes from a Randomized-Withdrawal Study of Long-Term Lisdexamfetamine Dimesylate Treatment in Children and Adolescents with Attention-Deficit/Hyperactivity Disorder
}

\author{
Tobias Banaschewski • Mats Johnson • Michel Lecendreux • \\ Alessandro Zuddas • Ben Adeyi • Paul Hodgkins • \\ Liza A. Squires • David R. Coghill \\ Published online: 20 August 2014 \\ (C) The Author(s) 2014. This article is published with open access at Springerlink.com
}

\begin{abstract}
Background The stimulant prodrug lisdexamfetamine dimesylate (LDX) is an effective and generally well tolerated treatment for the symptoms of attention-deficit/ hyperactivity disorder (ADHD). Positive impacts of LDX on health-related quality of life and functional impairment have previously been demonstrated in a 7-week, randomized, double-blind, placebo-controlled, phase III study in children and adolescents in Europe. Maintenance of these broad benefits, as well as symptomatic control, is a key goal of long-term management of ADHD.

Objective Secondary objectives of this multinational study in children and adolescents with ADHD were to assess the long-term maintenance of effectiveness of LDX in improving health-related quality of life and reducing
\end{abstract}

ClinicalTrials.gov identifier: NCT00784654; Shire study ID: SPD489-326.

Electronic supplementary material The online version of this article (doi:10.1007/s40263-014-0193-z) contains supplementary material, which is available to authorized users.

T. Banaschewski ( $\square)$

Department of Child and Adolescent Psychiatry and

Psychotherapy, Central Institute of Mental Health, Medical

Faculty Mannheim, University of Heidelberg, P.O. Box: 1221

20, 68072 Mannheim, Germany

e-mail: tobias.banaschewski@zi-mannheim.de

M. Johnson

Child Neuropsychiatry Unit, Queen Silvia Children's Hospital,

Gothenburg, Sweden

\section{Lecendreux}

Paediatric Sleep Centre and National Reference Centre for Orphan Diseases: Narcolepsy, Idiopathic Hypersomnia and Kleine-Levin Syndrome, Robert-Debré University Hospital, Paris, France functional impairment, as gauged using the Child Health and Illness Profile-Child Edition: Parent Report Form (CHIP-CE: PRF) and the Weiss Functional Impairment Rating Scale-Parent Report (WFIRS-P), respectively.

Methods Patients aged 6-17 years with diagnosed ADHD and a baseline ADHD Rating Scale IV total score of at least 28 were enrolled from the previous European study and from US sites. Patients who completed an open-label LDX treatment period of at least 26 weeks were randomized (1:1) to continue on their optimized dose of LDX or to switch to placebo for a 6-week, double-blind, withdrawal period. Parents completed CHIP-CE: PRF and WFIRS-P questionnaires at weeks 0,8 and 26 of the open-label period and at weeks 0 and 6 of the randomized-withdrawal period, or at early termination. The endpoint of each period was defined as the last visit with valid data. Effect sizes were the difference (LDX minus placebo) in least-squares (LS)-mean change from baseline to endpoint divided by root-mean-square error. $P$ values were nominal and not adjusted for multiple comparisons.

Results The open-label and randomized full analysis sets comprised 262 and 153 (LDX $n=76$; placebo $n=77$ )

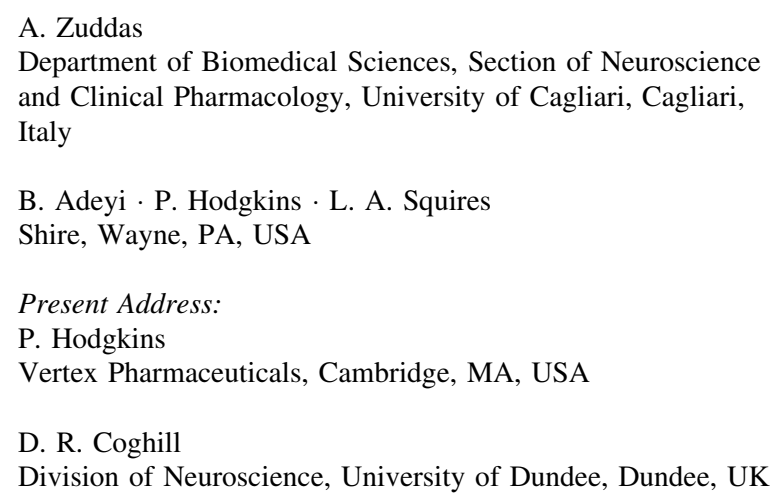


patients, respectively. Mean pretreatment CHIP-CE: PRF Tscores were more than one standard deviation below the normative mean in four of the five domains, and there was significant improvement across all domains from baseline to endpoint of the open-label period. In the randomized-withdrawal period, LS-mean CHIP-CE: PRF T-scores deteriorated in all domains in the placebo group, but not in the LDX group. Compared with placebo, the effect of LDX was significant in the Risk Avoidance (effect size 0.829; $p<0.001$ ), Achievement $(0.696 ; p<0.001)$ and Satisfaction $(0.636 ; p<0.001)$ domains. Mean pretreatment WFIRS-P scores were lowest in the Family domain and the Learning and School domain. WFIRS-P total score and scores in all domains improved significantly from baseline to endpoint of the open-label period. In the randomized-withdrawal period, LS-mean scores deteriorated in the placebo group but not in the LDX group. Compared with placebo, the effect of LDX was significant in the Family, Learning and School, and Risky Activities domains and in total (effect size 0.908; $p<0.001$ ).

Conclusions Using parent-rated instruments, long-term maintenance of the beneficial effect of LDX in multiple domains of health-related quality of life and functional impairment was demonstrated by comparison of treatment continuation and withdrawal under randomized, doubleblind, placebo-controlled conditions.

\section{Key Points}

Improved health-related quality of life (HRQoL) and reduced functional impairment in domains such as educational achievement and interpersonal relations are key goals in the management of attention-deficit/ hyperactivity disorder (ADHD), in addition to the relief of symptoms. The stimulant prodrug lisdexamfetamine dimesylate (LDX) has been shown to be an effective treatment for ADHD in children, adolescents and adults in short-term clinical trials.

This study used a randomized-withdrawal design to evaluate the impact of continued long-term (at least 26 weeks') LDX treatment of children and adolescents with ADHD on parental perceptions of HRQoL and functional impairment, using the Child Health and Illness Profile-Child Edition: Parent Report Form (CHIP-CE: PRF) and the Weiss Functional Impairment Rating Scale-Parent Report (WFIRS-P) instruments, respectively.

During the 6-week, placebo-controlled, doubleblind, randomized-withdrawal period, the effect of continued LDX treatment differed statistically significantly from placebo in three of the five CHIPCE: PRF domains, and in three of the six WFIRS-P domains and in WFIRS-P total score.

\section{Introduction}

Amfetamine- and methylphenidate-based psychostimulants are established treatments for individuals with attentiondeficit/hyperactivity disorder (ADHD). Lisdexamfetamine dimesylate (LDX), the first long-acting stimulant prodrug, undergoes rate-limiting enzymatic bioconversion to $d$-amfetamine in the bloodstream [1] and provides a daily duration of efficacy of at least $13 \mathrm{~h}$ post-dose in children and $14 \mathrm{~h}$ in adults, as determined in laboratory school and simulated workplace studies [2, 3]. The efficacy of oncedaily LDX in relieving the symptoms of ADHD compared with placebo has been demonstrated in a series of 4-week US-based clinical trials in children [4], adolescents [5] and adults [6], and more recently in a 7-week European study in children and adolescents (SPD489-325) [7]. Continuation of long-term LDX treatment has been shown to be more effective than placebo in maintaining efficacy in studies using randomized-withdrawal designs: a US study in adults with ADHD [8] and the present multinational study (SPD489-326) in children and adolescents with ADHD [9]. Such designs allow the value of continued treatment to be investigated more rigorously than in openlabel studies [10].

Although symptoms are themselves important, the broader impact of ADHD on day-to-day functioning and health-related quality of life (HRQoL) in domains such as educational achievement and interpersonal relations, often underlies a patient's or parent's decision to seek treatment [11]. Furthermore, the new guidance on severity of ADHD in the recent Fifth Edition of the 'Diagnostic and Statistical Manual of Mental Disorders' [12] states that severe ADHD is characterized either by intense symptoms or by marked impairment in social or occupational functioning. In study SPD489-325, beneficial effects of LDX were demonstrated on HRQoL and functional impairment, as gauged using the Child Health and Illness Profile-Child Edition: Parent Report Form (CHIP-CE: PRF) and the Weiss Functional Impairment Rating Scale-Parent Report (WFIRS-P) instruments, respectively [13]. The CHIP-CE: PRF is a generic (i.e. not disease-specific) measure of a child's overall health status in five domains (Achievement, Risk Avoidance, Satisfaction, Resilience and Comfort). Its validity and reliability in children and adolescents with ADHD have been established [14, 15]. The WFIRS-P is an ADHD-specific instrument designed around typical day-today problems, and has demonstrated internal consistency, sensitivity to change and convergent validity with other instruments [16, 17]. It provides measures of impairment in six domains (Family, Learning and School, Life Skills, Child's Self-Concept, Social Activities and Risky Activities) and overall (total score). LDX treatment was associated with significant placebo-adjusted improvements from 
baseline to endpoint of study SPD489-325 in the CHIP-CE: PRF domains of Achievement, Risk Avoidance, Resilience and Satisfaction, and in WFIRS-P total score and the domains of Learning and School, Family, Social Activities and Risky Activities [13].

With the aim of evaluating the long-term maintenance of efficacy of LDX, the subsequent study, SPD489-326, comprised open-label LDX treatment for at least 26 weeks with subsequent randomization to continue on LDX or withdraw to placebo for 6 weeks under double-blind conditions [9]. In the primary efficacy outcome, the treatment failure criteria (defined below) were met by a statistically significantly smaller proportion of patients in the LDX group than in the placebo group (15.8 vs $67.5 \%$, a difference of 51.7 [ $95 \%$ confidence interval (CI) 38.5, 65.0]; $p<0.001)$. This paper now reports the effects of long-term LDX treatment and its subsequent continuation or withdrawal on HRQoL and functional impairment outcomes from SPD489-326 (ClinicalTrials.gov identifier: NCT00784654).

\section{Methods}

The study procedures of SPD489-326 have previously been described in detail [9]. The study was conducted in accordance with the International Conference on Harmonisation of Good Clinical Practice, the Declaration of Helsinki (including its amendments), local ethical and legal requirements, and other currently applicable regulations [9]. The protocol was approved by an independent ethics committee/institutional review board and regulatory agency in each centre (as appropriate). Each patient's parent or legal guardian provided written, informed consent, and assent was obtained from each patient (if permitted by the site institutional review board or independent ethics committee) before commencing study-related procedures.

\subsection{Study Design}

Study SPD489-326 comprised an open-label period of at least 26 weeks' treatment with LDX, followed by a 6-week, double-blind, parallel-group, randomized-withdrawal period, in which patients continued to receive LDX or switched to placebo (Fig. 1). The study was originally designed as a 52-week, open-label extension of the previous European, phase III study (SPD489-325) [7], but the protocol was amended after study initiation in response to revised European Medicines Agency guidelines [18]. To ensure an adequate sample size for assessment of the primary efficacy outcome in the randomized-withdrawal period, additional patients were recruited from new study sites in the USA [9]. The study took place from January 2009 until October 2011.

\subsection{Patients}

The study was conducted at 37 sites in eight European countries and at four sites in the USA. Eligible patients were children (aged 6-12 years) and adolescents (aged 13-17 years) who met 'Diagnostic and Statistical Manual of Mental Disorders, Fourth Edition, Text Revision' (DSMIV-TR) criteria for a diagnosis of ADHD and who had an investigator-rated ADHD Rating Scale IV (ADHD-RS-IV) total score of 28 or higher at baseline, indicating symptoms of at least moderate severity.

Key exclusion criteria for all patients included failure to respond to previous osmotic-release oral system methylphenidate (OROS-MPH) therapy, and a comorbid psychiatric diagnosis with significant symptoms (not including oppositional defiant disorder). Patients whose current ADHD medication provided effective control of symptoms with acceptable tolerability were also excluded. For US patients only, previous therapy with LDX, previous participation in an LDX study or previous failure to respond to more than one adequate course of amfetamine therapy were additional exclusion criteria. European patients from the previous phase III study (SPD489-325) were eligible for the present study if they had received treatment with LDX, OROS-MPH or placebo for at least 4 weeks in SPD489325 , had attended both the week 4 and post-treatment washout study visits, had not experienced adverse events that would preclude exposure to LDX, and had not discontinued SPD489-325 owing to adverse events or noncompliance (defined as failure to take $80-100 \%$ of medication issued). US patients completed a 1- to 6-week washout period as part of SPD489-326.

\subsection{Drug Administration}

The open-label period comprised dose-optimization (4 weeks), dose-maintenance ( $\geq 20$ weeks) and fixed-dose (2 weeks) phases (Fig. 1). Instructions were that study drugs should be taken daily upon waking at approximately 07:00, beginning on the day after visit 1 (week 0). Patients initially received LDX $30 \mathrm{mg} /$ day; during dose optimization, daily doses could be increased or decreased in $20 \mathrm{mg}$ steps (maximum $70 \mathrm{mg}$; minimum $30 \mathrm{mg}$ ) until an 'acceptable response' was achieved (defined as a reduction of $\geq 30 \%$ in ADHD-RS-IV total score from baseline and a Clinical Global Impressions-Improvement [CGI-I] rating of 1 or 2, with tolerable side effects). Subsequent dose adjustments based on efficacy and tolerability were permitted in the dose-maintenance phase of the open-label period, but not in the fixed-dose phase (visits $1 \mathrm{R}-3 \mathrm{R}$ ) or 

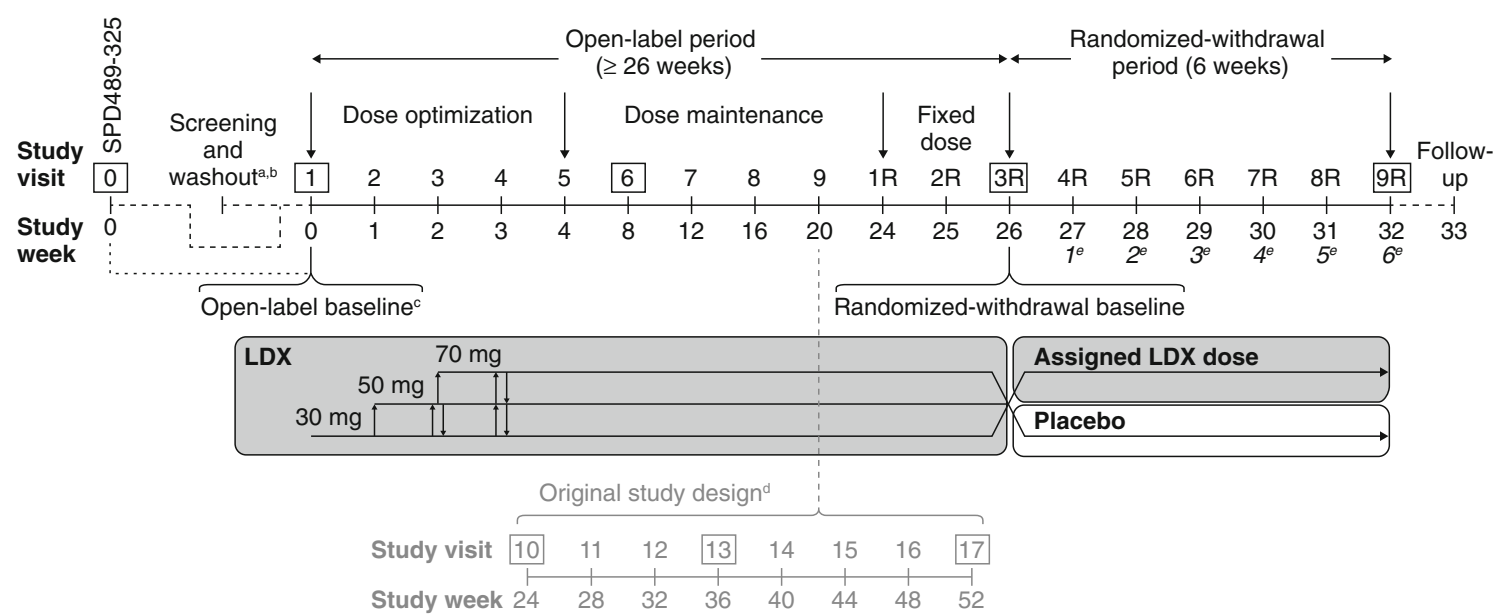

Fig. 1 SPD489-326 study design. CHIP-CE: PRF and WFIRS-P assessments were performed at the visits shown boxed, and/or at an early termination visit attended by patients who discontinued the study. ${ }^{a}$ For directly recruited patients only; other patients completed screening and washout as part of SPD489-325, the previous European, phase III study. ${ }^{\mathrm{b}}$ Screening and washout occurred 1-6 weeks before visit 1 for directly recruited patients. ${ }^{\mathrm{c}}$ See Table 1 for definitions of baseline and endpoint in the open-label and randomized-withdrawal periods. ${ }^{\mathrm{d}}$ Extended dose-maintenance phase for patients enrolled under the original protocol. These patients may have attended one or more additional visits (shown in grey) before entering the fixed-dose period. ${ }^{\mathrm{e}}$ Italicized numbers show weeks of the randomized-withdrawal period. CHIP-CE: PRF Child Health and Illness Profile-Child Edition: Parent Report Form, $R$ revised, WFIRS$P$ Weiss Functional Impairment Rating Scale-Parent Report thereafter. Patients enrolled before study protocol amendment underwent an extended dose-maintenance phase of open-label treatment for up to 52 weeks (Fig. 1).

At the end of the open-label period (visit 3R), subjects were randomized (1:1) to continue on their fixed, optimized dose of LDX or to receive placebo for the 6-week, doubleblind period (visits 3R-9R). Details of randomization and blinding have been described previously [9].

\subsection{Specific Continuation and Discontinuation Criteria}

Responder status at a stable dose was confirmed in the final, 2-week, fixed-dose phase of the open-label period, during which patients with either an ADHD-RS-IV total score above 22 or a CGI-S score of 3 or more, and those who experienced unacceptable tolerability as judged by investigators, discontinued the study. During the randomized-withdrawal period, patients with both an increase of at least $50 \%$ in ADHD-RSIV total score and an increase of two or more points in CGI-S score met the treatment failure criteria (primary efficacy outcome measure) and immediately discontinued the study. Patients who discontinued for any reason attended an early termination visit, which included assessments scheduled for the last on-treatment study visit (9R).

\subsection{HRQoL Measure: CHIP-CE: PRF}

Parents used a 5-point Likert-type response format to assess each of the 76 CHIP-CE: PRF questionnaire items, most of which refer to the past 4 weeks (the remainder are not associated with a specific time period). Data were handled according to the 'CHIP-CE Technical Manual' [19]: scores in the five domains and twelve associated subdomains were the mean of the component individual item scores (omitting items with a missing response; no more than $30 \%$ of the relevant items could be missing for a domain or subdomain score to be considered valid); and the domain and subdomain scores were standardized to $T$-scores with a mean of 50 and a standard deviation (SD) of 10. The algorithm for this standardization is based on normative data from community samples in the USA [15]. A $T$-score of 43 or below indicates poor HRQoL in a domain or subdomain; a $T$-score of 57 or above indicates excellent HRQoL [20].

\subsection{Functional Impairment Measure: WFIRS-P}

Parents used a Likert-type scale ( $0-3$ or 'not applicable') to assess each of the 50 WFIRS-P questionnaire items, all of which refer to the previous 4 weeks. The WFIRS-P scores for each patient were the mean of the item scores in each domain, or of all the item scores for total score (omitting items with a missing response; no more than $30 \%$ of the relevant items could be missing for a domain or total score to be considered valid). Higher WFIRS-P scores indicate more severe functional impairment. At present, there are no published reference population data for the WFIRS-P. 


\subsection{Schedule of Assessments}

Parents (or legally authorized representatives) completed CHIP-CE: PRF and WFIRS-P questionnaires at the study visits indicated in Fig. 1, and/or at early termination. Baseline and endpoint of the open-label period and baseline and endpoint of the randomized-withdrawal period were defined as shown in Table 1.

\subsection{Statistical Analyses}

Statistical analyses were based on the open-label full analysis set, defined as all patients who received at least one dose of study drug, and on the randomized full analysis set, defined as all patients who were randomized and received at least one dose of study drug during the randomizedwithdrawal period. Both of these sets excluded 14 patients from one site where there were violations of good clinical practice [9].

The CHIP-CE: PRF and WFIRS-P were pre-specified as secondary efficacy outcome measures. Sample size was determined as previously reported and was based on the primary efficacy outcome of treatment failure during the randomized-withdrawal period [9]. The study was not powered for statistical analysis of any secondary outcome. No adjustment of $p$ values for multiple comparisons was pre-specified or performed for secondary efficacy variables. Quoted $p$ values are descriptive rather than inferential, and those below 0.05 therefore represent nominal statistical significance. Results were analysed using $\mathrm{SAS}^{\circledR}$ Version 9.1.3 or higher (SAS Institute Inc., Cary, NC, USA).

For the open-label period, the changes in CHIP-CE: PRF domain and subdomain $T$-scores or WFIRS-P total and domain scores at endpoint were compared with open-label baseline using one-sample, two-sided $t$ tests at a significance level of 0.05 . Changes from open-label baseline to other study visits were not analysed statistically. This approach to analysis of open-label period data was prespecified in the study protocol.

For the randomized-withdrawal period, the changes from baseline to endpoint in CHIP-CE: PRF domain and subdomain $T$-scores or WFIRS-P total and domain scores were compared between the LDX and placebo groups using analysis of covariance models at the 0.05 significance level, with treatment group as the effect of interest, the corresponding baseline score as the covariate, and country and age group as blocking factors. These between-group statistical comparisons were pre-specified in the study protocol; within-group comparisons of changes at endpoint versus baseline from the same models were ad hoc analyses and were not pre-specified. The effect size (standardized mean difference) for each domain or subdomain $T$-score (CHIP-CE: PRF) or domain or total score (WFIRS-P) was based on the difference (LDX minus placebo) in leastsquares (LS)-mean change from baseline to endpoint divided by root-mean-square error. This is analogous to an effect size based on Cohen's $d$ or Hedges' $g$ (difference in means divided by pooled standard deviation).

\section{Results}

\subsection{Patient Disposition and Baseline Characteristics}

The open-label full analysis set comprised 262 patients: 222 from the previous European, phase III study (SPD489325 ) and 40 from the additional US sites. Of these, 153

Table 1 Definitions of baseline and endpoint in the two study periods

$\begin{array}{lc}\begin{array}{l}\text { Open-label baseline } \\ \begin{array}{l}\text { Patients recruited from SPD489-325 in Europe } \\ (n=222)\end{array}\end{array} & \begin{array}{c}\text { Visit } 0 \text { (week 0) of SPD489-325 (after screening and washout as part of SPD489-325) } \\ \text { Additional US patients }(n=40) \\ \text { Visit } 1 \text { (week 0) of the present study (after screening and washout as part of the present } \\ \text { study) }\end{array} \\ \begin{array}{l}\text { Open-label endpoint } \\ \text { Patients under the original protocol }\end{array} & \begin{array}{c}\text { The last on-treatment visit with valid data, up to and including visit 17 (week 52), or early } \\ \text { termination }\end{array} \\ \text { Patients who switched protocols } & \begin{array}{c}\text { The last on-treatment visit with valid data, up to and including visit 3R (week } \geq 26 \text { ), or } \\ \text { early termination }\end{array} \\ \text { Patients under the revised protocol } & \begin{array}{c}\text { The last on-treatment visit with valid data, up to and including visit 3R (week 26), or early } \\ \text { termination } \\ \text { Visit 3R (week } 0 \text { of the randomized-withdrawal period) }\end{array} \\ \text { Randomized-withdrawal baseline } & \text { Visit 9R (week } 6 \text { of the randomized-withdrawal period) or early termination } \\ \text { Randomized-withdrawal endpoint } & \end{array}$

See Fig. 1 for schedule of study visits. Numbers are those in the full analysis set. SPD489-325 was the previous 7-week, European, phase III study of LDX

$R$ revised 
completed the amended, open-label period of at least 26 weeks and 7 completed the original, open-label period of 52 weeks. In the open-label full analysis set, the mean age of patients was 10.8 years (SD 2.73 years), $76.3 \%$ were male, and their mean ADHD-RS-IV total score at open-label baseline was 40.6 (SD 6.83). Further patient characteristics at open-label baseline are shown in Supplementary Table A; additional information on patient disposition and the study populations has been reported previously [9].

All 153 patients who completed the amended ( $\geq 26$ week) open-label period entered the randomized withdrawal period and were included in the randomized full analysis set (LDX 76; placebo 77). Of these patients, 132 were recruited from SPD489-325 and 21 from the US sites; and 57 in the LDX group and 16 in the placebo group completed the randomized-withdrawal period. The mean age of patients in the randomized full analysis set was 11.1 years (SD 2.59 years) and $77.8 \%$ were male (Supplementary Table A). At randomized-withdrawal baseline, mean ADHD-RS-IV total scores were 10.2 (SD, 5.92) in the LDX group and 9.5 (6.45) in the placebo group [9].

\subsection{HRQoL Outcomes: CHIP-CE: PRF}

\subsubsection{CHIP-CE: PRF T-Scores at Open-Label Baseline}

At open-label baseline, the lowest mean CHIP-CE: PRF Tscore (indicating the most profound HRQoL deficit) was observed in the Achievement domain (mean 30.2; SD 9.71), with lower mean $T$-scores in the associated Academic Performance subdomain than in Peer Relations (Fig. 2a). Mean baseline $T$-scores were also more than one population SD (10 points) below the normative mean (50 points) in the Risk Avoidance, Satisfaction and Resilience domains, but not in the Comfort domain and in its associated subdomains, with the exception of Emotional Comfort (Fig. 2a). Corresponding medians and ranges are given in Supplementary Table B.

\subsubsection{CHIP-CE: PRF T-Scores in the Open-Label Period}

Mean increases (improvements) in CHIP-CE: PRF $T$ scores from baseline to endpoint of the open-label period were significant in all domains and were greatest in Risk Avoidance, followed by Achievement, Satisfaction, Comfort and Resilience (Fig. 2a). In all domains, most of the increase in mean domain $T$-scores during the open-label period was evident by visit 6 (week 8 ), although mean domain $T$-scores were higher at visit $3 \mathrm{R}$ ( $\geq$ week 26 ) than at visit 6 (week 8). At visit $3 \mathrm{R}, T$-scores were within one $\mathrm{SD}$ of the normative mean (i.e. above 40) in all domains, including those which showed profound HRQoL deficits at baseline (Fig. 2b).

\subsubsection{CHIP-CE: PRF T-Scores in the Randomized- Withdrawal Period}

At baseline of the randomized-withdrawal period (visit 3R), mean CHIP-CE: PRF domain and subdomain $T$-scores were similar in the groups allocated to continue LDX or withdraw to placebo (Table 2). Corresponding medians and ranges are given in Supplementary Table B.

CHIP-CE: PRF $T$-scores in patients who continued on LDX did not statistically significantly change from baseline to endpoint in all domains and subdomains, with the exception of significant LS-mean increases (improvements) in the Satisfaction domain and the associated Satisfaction with Self subdomain (Fig. 3). Withdrawal to placebo for up to 6 weeks was associated with significant LS-mean decreases (deteriorations) in CHIP-CE: PRF $T$-scores in all domains from baseline to endpoint (Fig. 3). Within the Achievement and Risk Avoidance domains, decreases were significant in all associated subdomains; within the remaining CHIP-CE: PRF domains, decreases were significant only in the Social Problem-Solving, Satisfaction with Health and Emotional Comfort subdomains.

Differences between the LDX and placebo groups in the LS-mean changes from baseline to endpoint were significant $(p<0.001)$ for the Risk Avoidance (effect size 0.829), Achievement (effect size 0.696) and Satisfaction (effect size 0.636) domains and in all of the subdomains within these domains (Fig. 3). Within the Resilience and Comfort domains, there were significant differences $(p<0.01)$ between LDX and placebo in the Social Problem-Solving and Emotional Comfort subdomains only, with effect sizes of 0.480 and 0.581 , respectively (Fig. 3).

At endpoint in the placebo group, mean $T$-scores had fallen to more than one SD below the normative mean in two domains (Achievement and Satisfaction) and three subdomains, but in no domain or subdomain in the LDX group (Table 2). Corresponding medians and ranges are given in Supplementary Table B.

\subsection{Functional Impairment Outcomes: WFIRS-P}

\subsubsection{WFIRS-P Scores at Open-Label Baseline}

At open-label baseline, WFIRS-P scores were highest (indicating greatest impairment) in the Family domain and the Learning and School domain, with mean scores of 1.36 (SD 0.750) and 1.28 (0.612), respectively (Fig. 4a). Corresponding medians and ranges are given in Supplementary Table C. 
Fig. 2 CHIP-CE: PRF T-scores during the open-label period $(n=262)$. a Mean $T$-scores at open-label baseline and histogram showing mean change from open-label baseline to endpoint for CHIP-CE: PRF domains and subdomains. Error bars show $95 \%$ CIs. Positive changes indicate improved HRQoL. *** $p<0.001$,

$* * p<0.01$, change at endpoint versus baseline (two-sided $t$ test). $P$ values are nominal and were not adjusted for multiple comparisons. b Mean CHIP-CE: PRF domain $T$-scores at baseline (BL), endpoint (EP), visit 6 (V6 8 weeks) and visit $3 R$ (V3R $\geq 26$ weeks) of the open-label period. Error bars show $95 \%$ confidence intervals. These data were not subjected to statistical testing. Numbers of observations ( $n$ ) are shown for each data point. A $T$-score of 43 or below indicates poor HRQoL in a domain or subdomain; a $T$ score of 57 or above indicates excellent HRQoL [20]. The horizontal axis is positioned at the normative mean ( $T$-score of 50). BL baseline, $C H I P-C E$ :

$P R F$ Child Health and Illness Profile-Child Edition: Parent Report Form, $C I$ confidence interval, $E P$ endpoint, $H R Q o L$ health-related quality of life, $R$ revised, $S D$ standard deviation, $V$ visit

\section{(a)}
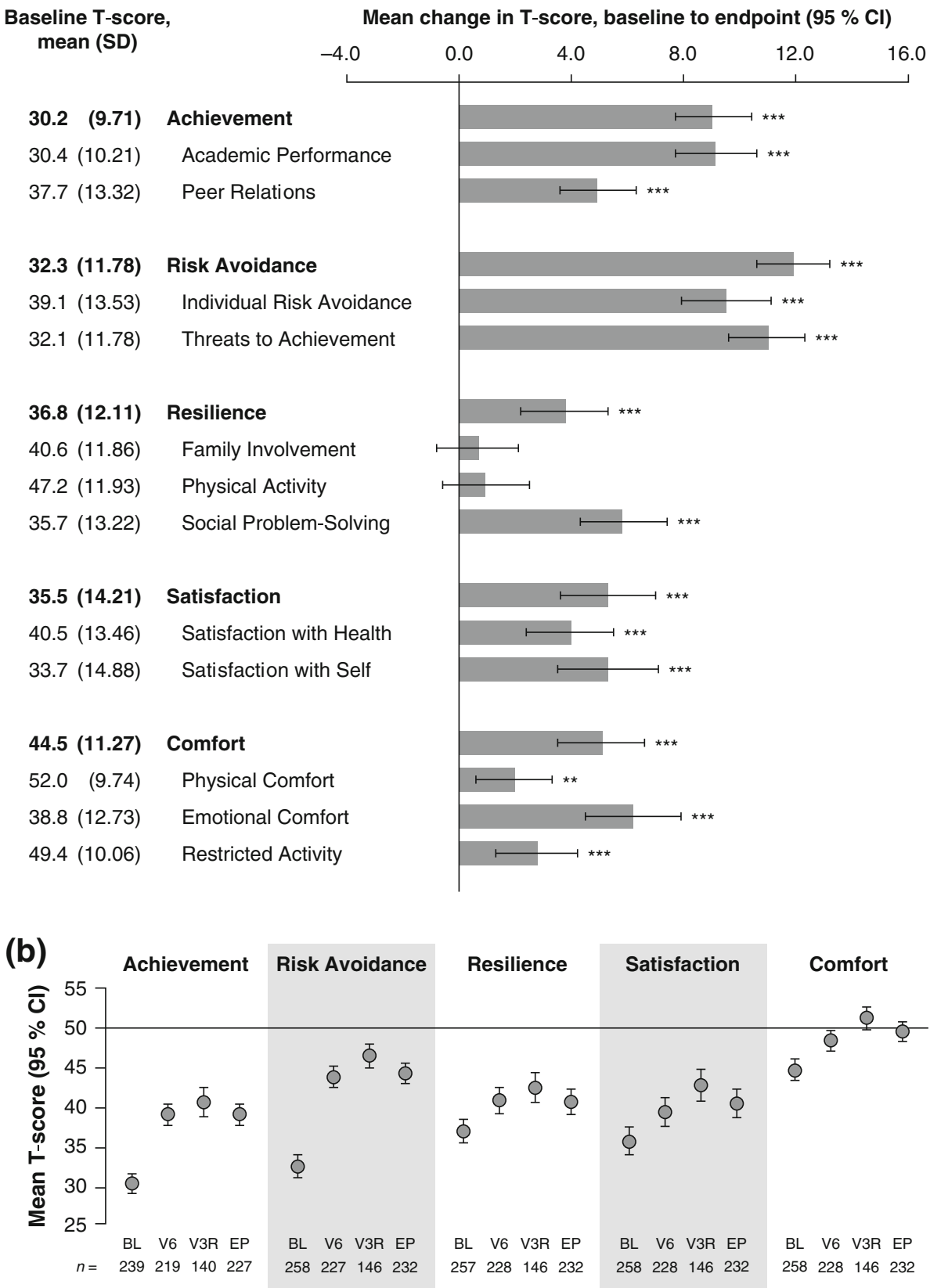

\subsubsection{WFIRS-P Scores in the Open-Label Period}

WFIRS-P domain and total scores significantly decreased (indicating improvement) from baseline to endpoint of the open-label LDX treatment period (Fig. 4a). The mean changes in score were largest in the Family domain and the Learning and School domain. In all domains, and in total, most of the decrease in WFIRS-P score during the openlabel period was evident by visit 6 (week 8), although mean domain and total scores were lower in all domains at visit $3 \mathrm{R}$ ( $\geq$ week 26) than at visit 6 (week 8) (Fig. 4b).

\subsubsection{WFIRS-P Scores in the Randomized-Withdrawal Period}

At baseline of the randomized-withdrawal period (visit 3R), mean WFIRS-P domain and total scores were lower in the group allocated to placebo than in the group allocated to LDX (Table 3; data not analysed statistically). Corresponding medians and ranges are given in Supplementary Table C.

WFIRS-P scores did not significantly change in any domain or in total in the LDX group from baseline to 
Table 2 Randomized-withdrawal period: mean (SD) CHIP-CE: PRF $T$-scores at baseline and endpoint $(n=153)$

\begin{tabular}{|c|c|c|c|c|}
\hline & \multicolumn{2}{|l|}{ Baseline } & \multicolumn{2}{|l|}{ Endpoint } \\
\hline & $\operatorname{LDX}(68 \leq n \leq 73)$ & Placebo $(71 \leq n \leq 73)$ & $\operatorname{LDX}(59 \leq n \leq 62)$ & Placebo $(62 \leq n \leq 65)$ \\
\hline Achievement & $39.6(10.58)$ & $41.2(11.11)$ & $40.1(11.70)$ & $35.3(11.63)$ \\
\hline Academic Performance & $40.7(11.42)$ & $41.1(10.14)$ & $40.6(13.46)$ & $34.4(12.41)$ \\
\hline Peer Relations & $42.0(10.77)$ & $45.3(12.13)$ & $43.1(11.56)$ & $42.4(12.84)$ \\
\hline Risk Avoidance & $45.8(9.08)$ & $46.8(9.11)$ & $47.5(9.16)$ & $41.3(10.53)$ \\
\hline Individual Risk Avoidance & $50.3(10.02)$ & $51.0(10.08)$ & $52.7(8.66)$ & $47.7(11.54)$ \\
\hline Threats to Achievement & $44.4(9.08)$ & $45.4(9.04)$ & $45.5(10.13)$ & $39.8(10.08)$ \\
\hline Resilience & $42.0(11.56)$ & $42.6(11.87)$ & $43.3(11.25)$ & $40.2(13.79)$ \\
\hline Family Involvement & $43.2(12.46)$ & $41.5(12.46)$ & $43.2(10.74)$ & $40.3(14.73)$ \\
\hline Physical Activity & $47.7(11.57)$ & $49.1(10.85)$ & $48.2(11.72)$ & $49.4(11.81)$ \\
\hline Social Problem-Solving & $43.0(11.05)$ & $44.6(12.11)$ & $45.1(11.71)$ & $40.9(11.90)$ \\
\hline Satisfaction & $41.9(11.40)$ & $43.3(13.19)$ & $44.9(11.88)$ & $39.3(14.83)$ \\
\hline Satisfaction with Health & $45.8(10.90)$ & $46.1(13.13)$ & $48.0(11.89)$ & $42.6(13.88)$ \\
\hline Satisfaction with Self & $39.6(12.51)$ & $42.1(11.28)$ & $42.6(10.67)$ & 38.4 (12.95) \\
\hline Comfort & $51.0(8.43)$ & $51.1(8.92)$ & $51.1(9.32)$ & $48.5(9.94)$ \\
\hline Physical Comfort & $55.9(6.53)$ & $53.2(8.75)$ & $54.3(8.04)$ & $53.6(9.11)$ \\
\hline Emotional Comfort & $45.7(10.37)$ & $48.0(10.38)$ & $47.9(9.76)$ & $44.2(11.15)$ \\
\hline Restricted Activity & $52.5(8.11)$ & $52.7(6.91)$ & $51.0(10.48)$ & $50.0(9.50)$ \\
\hline
\end{tabular}

See Table 1 for definitions of baseline and endpoint. A $T$-score of 43 or below indicates poor HRQoL in a domain or subdomain; a $T$-score of 57 or above indicates excellent HRQoL [20]. See Supplementary Table A for corresponding medians and ranges. Numbers of observations $(n)$ for each domain or subdomain were in the ranges indicated and varied depending on data validity. These data were not analysed statistically

CHIP-CE: PRF Child Health and Illness Profile-Child Edition: Parent Report Form, LDX lisdexamfetamine dimesylate, SD standard deviation

endpoint of the randomized-withdrawal period, but significant LS-mean increases in WFIRS-P scores (indicating worsened functioning) were observed in all domains and in total in the placebo group (Fig. 5). The difference between the LDX and placebo groups in the LS-mean changes from baseline to endpoint was significant for total score $(p<0.001$, effect size 0.908$)$ and the Family $(p<0.001$, effect size 0.859), Learning and School $(p<0.001$, effect size 0.716$)$ and Risky Activities $(p<0.01$, effect size 0.506) domains.

At endpoint, mean total scores and scores in all the domains except Social Activities were higher in the placebo group than in the LDX group (Table 3). Corresponding medians and ranges are given in Supplementary Table C.

\section{Discussion}

Improved HRQoL and reduced functional impairment are key goals in the management of ADHD [21-23]. The present study evaluated the impact of continued long-term (at least 26 weeks') LDX treatment of children and adolescents with ADHD on parental perceptions of HRQoL and functional impairment, using the CHIP-CE: PRF and
WFIRS-P instruments, respectively. The effect of continued LDX treatment during the 6-week, placebo-controlled, double-blind, randomized-withdrawal period differed statistically significantly from placebo in three of the five CHIP-CE: PRF domains, and in three of the six WFIRS-P domains and in WFIRS-P total score. Following improvement in all domains of both instruments during open-label LDX treatment, HRQoL and functional impairment scores deteriorated in the placebo group, but not in the LDX group. At open-label baseline, mean CHIP-CE: PRF domain $T$-scores were more than one SD (10 points) below the normative mean (50) in four of the five domains, with Achievement exhibiting the greatest deficit, followed by Risk Avoidance, Satisfaction and Resilience. These results are concordant with published CHIP-CE: PRF data in untreated patients with ADHD in Europe [14, 24] and indicate that HRQoL is severely and pervasively compromised in children and adolescents with ADHD compared with the CHIP-CE: PRF reference population. A Spanish CHIP-CE: PRF reference population has been shown to be generally similar to the US reference population used for conversion of CHIP-CE: PRF responses to $T$-scores [25], indicating that the normalization procedure is likely to be applicable to the population enrolled in the present study. 


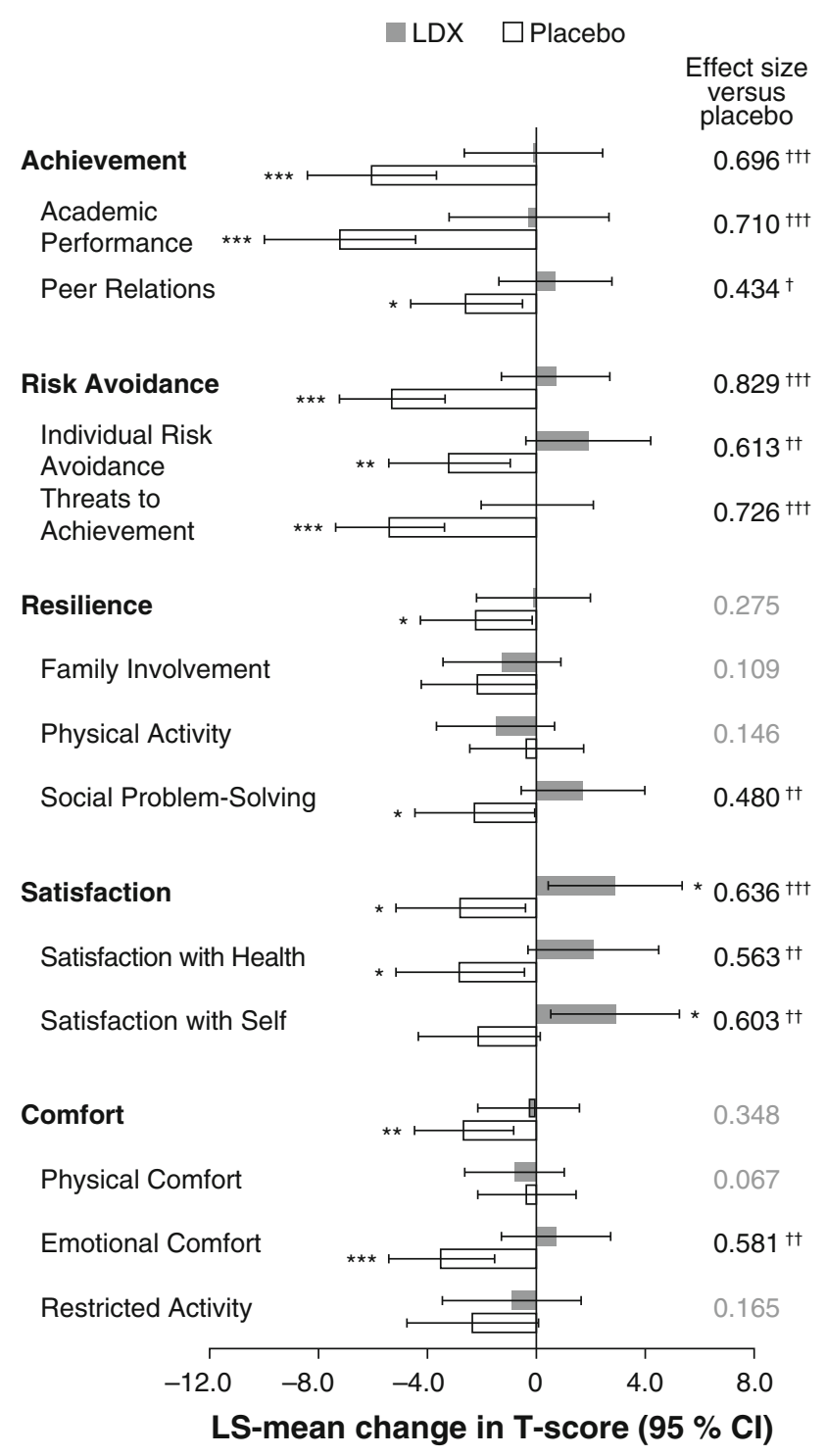

Fig. 3 Statistical analysis of changes in CHIP-CE: PRF $T$-scores from baseline to endpoint of the randomized-withdrawal period $(n=153)$. Histogram shows LS-mean change in CHIP-CE: PRF domain and subdomain $T$-scores from baseline to endpoint of the randomized-withdrawal period. Error bars show $95 \%$ CIs. Effect size is the difference in LS-mean change (LDX minus placebo) divided by root-mean-square error. Positive changes indicate improved health-related quality of life. ${ }^{*} p<0.05, * * p<0.01$, $* * * p<0.001$, change from baseline to endpoint (ad hoc analysis); ${ }^{\dagger} p<0.05,{ }^{\dagger} p<0.01,{ }^{\dagger \dagger} p<0.001$, LDX change versus placebo change (prespecified analysis). $P$ values are nominal and were not adjusted for multiple comparisons. CHIP-CE: PRF Child Health and Illness Profile-Child Edition: Parent Report Form, $L D X$ lisdexamfetamine dimesylate, $L S$ least-squares

Improvements in HRQoL during open-label LDX treatment for at least 26 weeks in the present study were most pronounced in the CHIP-CE: PRF domains with the most severe baseline deficits, namely Achievement and Risk Avoidance. These improvements occurred mainly during the first 8 weeks of treatment. Within the
Achievement domain, a smaller improvement from a higher baseline was observed in Peer Relations than in Academic Performance in the open-label period of the present study, in accordance with previous factor analyses indicating that these subdomains represent distinct constructs in the child and adolescent ADHD population [14, 24]. The HRQoL results from the open-label period of the present study are consistent with the pattern and magnitude of improvements in CHIP-CE: PRF domain and subdomain $T$-scores seen in the previous placebo-controlled, 7-week study (SPD489-325) [13].

The largest improvements in WFIRS-P scores during open-label LDX treatment were also observed in those domains of functional impairment with the worst scores at baseline (Family, and Learning and School). Improvements in WFIRS-P scores also occurred mainly during the first 8 weeks of treatment, and the pattern and magnitude of improvements in WFIRS-P domain scores in the open-label period of the present study were again consistent with that observed in the previous placebo-controlled, 7-week study (SPD489-325) [13]. These results and those of other studies [26-28] support the use of the WFIRS-P as a functional outcome measure in patients with ADHD. Interpretation of WFIRS-P scores is hampered, however, by the possibility (not unique to the WFIRS-P) that mean scores may conceal high individual item scores of potential clinical relevance, and by the lack of published psychometric properties and normative or reference population data. Although studies have been conducted to investigate these, the results have not yet been published in full [29].

The present study was the first to use a randomizedwithdrawal design to examine whether continuation of stimulant pharmacotherapy is required for maintained beneficial effects of medication on HRQoL and functional impairment in patients with ADHD. Compared with the deterioration in all domains of both instruments in patients who withdrew to placebo under double-blind conditions, maintained efficacy of LDX was observed in the CHIP-CE: PRF domains of Achievement, Risk Avoidance and Satisfaction (effect sizes 0.696, 0.829 and 0.636, respectively), in the WFIRS-P domains of Family, Learning and School, and Risky Activities (effect sizes 0.859, 0.716 and 0.506, respectively), and in WFIRS-P total score (effect size 0.908). Improvement from baseline to endpoint of the randomized-withdrawal period was observed only in the CHIP-CE: PRF Satisfaction domain, and only in patients who continued on their LDX treatment. Thus, the domains of HRQoL and functioning with the greatest deficits at baseline tended to exhibit both the greatest improvements during LDX treatment and the greatest deteriorations following LDX withdrawal.

There is no evidence that ADHD medications have any beneficial effect on the underlying neuropathophysiology 


\section{(a)}
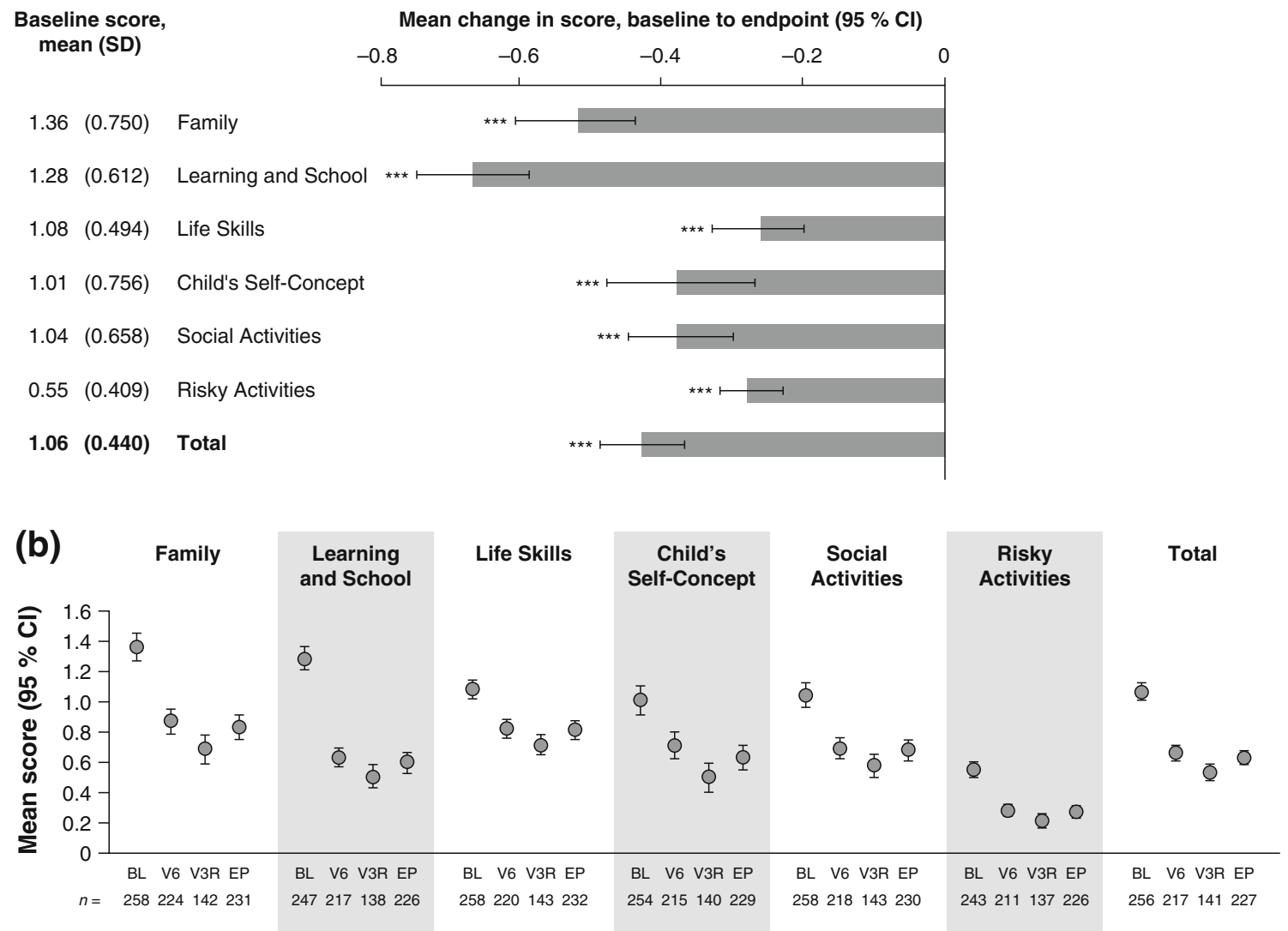

Fig. 4 WFIRS-P scores during the open-label period $(n=262)$. a Mean scores at open-label baseline and histogram showing mean change from open-label baseline to endpoint for WFIRS-P domain and total scores. Error bars show $95 \%$ confidence intervals. Negative changes indicate reduced functional impairment. $* * * p<0.001$, $* * p<0.01$, change at endpoint versus baseline (two-sided $t$ test). $P$ values are nominal and were not adjusted for multiple comparisons. b Mean WFIRS-P domain and total scores at baseline (BL), endpoint
(EP), visit 6 (V6 8 weeks) and visit $3 \mathrm{R}$ (V3R $\geq 26$ weeks) of the open-label period. Error bars show $95 \%$ CIs. These data were not subjected to statistical testing. Numbers of observations $(n)$ are shown for each data point. Higher scores indicate more severe functional impairment. $B L$ baseline, $C I$ confidence interval, $E P$ endpoint, $R$ revised, $S D$ standard deviation, $V$ visit, WFIRS- $P$ Weiss Functional Impairment Rating Scale-Parent Report

Table 3 Randomized-withdrawal period: mean (SD) WFIRS-P scores at baseline and endpoint $(n=153)$

\begin{tabular}{llllll}
\hline & \multicolumn{2}{l}{ Baseline } & & \multicolumn{2}{l}{ Endpoint } \\
\cline { 2 - 3 } & LDX $(68 \leq n \leq 73)$ & Placebo $(69 \leq n \leq 72)$ & & LDX $(59 \leq n \leq 63)$ & Placebo $(61 \leq n \leq 65)$ \\
\hline Family & $0.76(0.526)$ & $0.63(0.581)$ & $0.46(0.451)$ & $0.69(0.531)$ & $1.00(0.692)$ \\
Learning and School & $0.53(0.421)$ & $0.65(0.334)$ & $0.58(0.474)$ & $0.73(0.504)$ \\
Life Skills & $0.76(0.467)$ & $0.41(0.485)$ & $0.73(0.473)$ & $0.78(0.458)$ \\
Child's Self-Concept & $0.60(0.580)$ & $0.53(0.469)$ & $0.57(0.607)$ & $0.68(0.681)$ \\
Social Activities & $0.64(0.437)$ & $0.19(0.237)$ & $0.71(0.491)$ & $0.71(0.559)$ \\
Risky Activities & $0.23(0.215)$ & $0.48(0.315)$ & $0.22(0.232)$ & $0.30(0.319)$ \\
Total & $0.58(0.295)$ & $0.58(0.329)$ & $0.71(0.387)$ \\
\hline
\end{tabular}

See Table 1 for definitions of baseline and endpoint. Higher scores indicate more severe functional impairment. See Supplementary Table B for corresponding medians and ranges. Numbers of observations $(n)$ for each domain or total score were in the ranges indicated and varied depending on data validity. These data were not analysed statistically

LDX lisdexamfetamine dimesylate, SD standard deviation, WFIRS-P Weiss Functional Impairment Rating Scale-Parent Report 


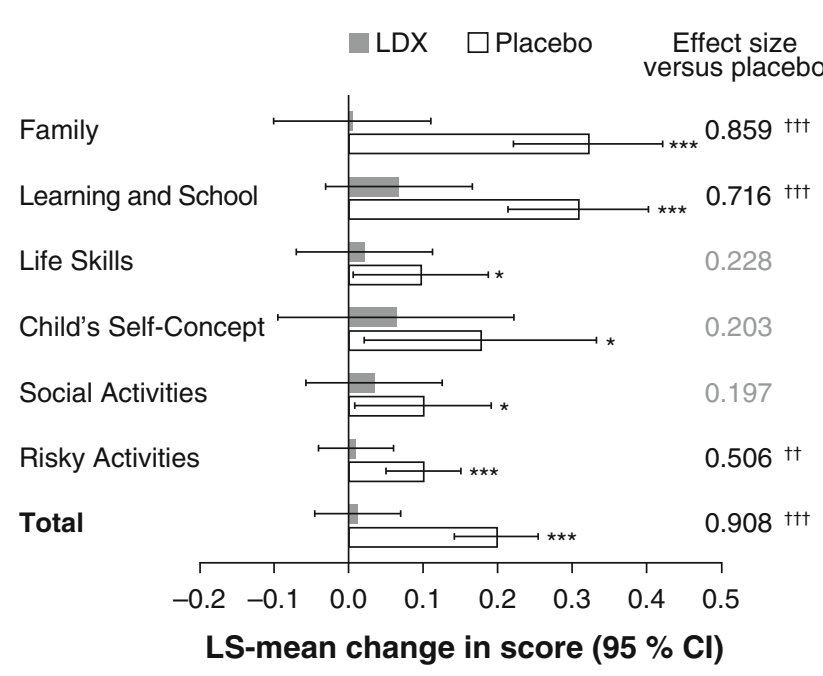

Fig. 5 Statistical analysis of changes in WFIRS-P scores from baseline to endpoint of the randomized-withdrawal period $(n=153)$. Histogram shows LS-mean change in WFIRS-P domain and total scores from baseline to endpoint of the randomized-withdrawal period. Error bars show $95 \%$ CIs. Effect size is the difference in LSmean change (LDX minus placebo) divided by root-mean-square error. Positive changes indicate worsened functional impairment. ${ }^{*} p<0.05, * * p<0.01, * * * p<0.001$, change from baseline to endpoint (ad hoc analysis); ${ }^{\dagger} p<0.05,{ }^{\dagger} p<0.01,{ }^{\dagger \dagger} p<0.001$, LDX change versus placebo change (prespecified analysis). $P$ values are nominal and were not adjusted for multiple comparisons. $L D X$ lisdexamfetamine dimesylate, $L S$ least-squares, WFIRS-P Weiss Functional Impairment Rating Scale-Parent Report

of the disorder. This is consistent with the previously reported rapid return of symptoms in patients who switched from LDX to placebo [9], and with the worsening of functional impairment and HRQoL described here. However, it is noteworthy that CHIP-CE: PRF and WFIRS-P scores in the placebo group at endpoint of the randomizedwithdrawal period did not return to pre-treatment levels at open-label baseline. It is possible that residual benefits of LDX therapy may have extended beyond the point of treatment withdrawal, or that underlying deficits in HRQoL and functioning may have attenuated over the course of the study (which involved repeated clinic visits). Alternatively, because patients were withdrawn from the study during the randomized-withdrawal period if they met the predefined treatment failure criteria that formed the basis of the primary efficacy outcome (both an increase of $\geq 50 \%$ in ADHD-RS-IV total score and an increase of $\geq 2$ points in CGI-S score) [9], intensity of symptoms, HRQoL deficits or functional impairment may have been underestimated at randomized-withdrawal endpoint. Finally, the repeated administration of the parent-rated questionnaires could also have resulted in underestimation at endpoint.

None of the CHIP-CE: PRF or WFIRS-P effect sizes for LDX continuation versus withdrawal to placebo were as large as the symptom-based ADHD-RS-IV total score effect size of 1.493 in the randomized-withdrawal period (based on mean changes from baseline to endpoint of 1.9 [SD 6.97] in the LDX group and 14.5 [9.95] in the placebo group, with a statistically significant $[p<0.001]$ least squares mean difference [LDX minus placebo] of -12.6 [95\% CI $-15.4,-9.8]$ ] [9]. Similarly, effect sizes were greater for the ADHD-RS-IV than for the CHIP-CE: PRF or WFIRS-P outcomes in the previous placebo-controlled, 7-week study (SPD489-325) [13]. In both studies, participants were recruited based on symptom severity and not on HRQoL or functional impairment criteria, but 'large' effect sizes $(>0.8$ [30]) were nevertheless observed using the CHIP-CE: PRF and WFIRS-P secondary outcome measures. These observations are consistent with the hypothesis that functional impairment and HRQoL are neither wholly dependent on, nor wholly independent of, ADHD symptoms, even in the domains that are most responsive to stimulant therapy [21-23, 31]. The concordance between the results of the present study and those of SPD489-325 suggests that the interrelationships of HRQoL and functional impairment with ADHD symptoms are broadly similar during treatment initiation and following withdrawal of long-term treatment. Whether symptoms of ADHD deteriorate more rapidly than domains of HRQoL or functioning following LDX withdrawal, and whether ADHD symptoms lead directly or indirectly to HRQoL deficits and functional impairment, are questions not addressed in the present study.

To our knowledge, only four other randomized-withdrawal studies of ADHD pharmacotherapies have been published [10], and only one of these deployed a measure of HRQoL or functional impairment. In that study, 416 children and adolescents with ADHD completed a 12-week open-label period of atomoxetine treatment and were then randomized to receive atomoxetine or placebo for up to 9 months under double-blind conditions. One of the secondary efficacy measures was the Child Health Questionnaire (CHQ), a measure of children's overall physical and psychosocial well-being. The authors did not state which version of the CHQ was used and did not report physical summary scores or individual 'concept' (i.e. domain) scores. Post-randomization CHQ psychosocial summary scores in the placebo group worsened to a significantly greater extent than in the atomoxetine group (mean change from baseline, -9.5 [SD 13.2] vs -5.6 [12.0]; $p=0.016$ ) [32].

The double-blind, placebo-controlled, randomizedwithdrawal design of the present long-term study is one of its key strengths, alongside the enrolment of both children and adolescents in Europe and the USA. The inclusion of both HRQoL and functional impairment as secondary 
efficacy outcomes is also a strength of the study design, even though the study population was enrolled based on symptom severity criteria. The strengths and weaknesses intrinsic to the WFIRS-P and CHIP-CE: PRF instruments have been previously discussed [13]. As mentioned above, the randomized-withdrawal endpoint included early termination assessments of patients who discontinued the study if they met the treatment failure criteria during the randomized-withdrawal period, and who were not assessed thereafter. Furthermore, the study design was such that patients who entered the randomized-withdrawal period were those who had benefited from, and were able to tolerate, LDX treatment during the open-label period. Given the rapid reappearance of symptoms upon LDX withdrawal, with a median time to treatment failure of 17.0 days $(95 \%$ CI 12.0, 22.0) in the placebo group [9], it is possible that some patients or parents may have drawn their own conclusions about the identity of the allocated randomized treatment, and that this may have affected scores on the parent-rated secondary efficacy measures [13]. Other advantages and disadvantages of the study design have been previously discussed [9].

\section{Conclusions}

Continued treatment with once-daily, optimized doses of LDX maintained the improvements in parentally perceived HRQoL and functional impairment acquired during at least 26 weeks of open-label treatment to a greater extent than placebo in this international, phase III, randomized-withdrawal study. Prolonged effectiveness was most pronounced in the CHIP-CE: PRF and WFIRS-P domains that correspond to clinically relevant treatment objectives, such as reducing risky behaviour, improving relationships with family and peers and improving academic achievement. These results may assist healthcare professionals in planning pharmacological treatment of children and adolescents with ADHD who face particular challenges in domains of HRQoL and day-to-day functioning.

Acknowledgments The authors thank the patients and investigators who took part in the study.

This study was funded by Shire Development LLC. Shire develops and markets drugs to treat psychiatric disorders, including ADHD.

Shire International $\mathrm{GmbH}$ provided funding to Oxford PharmaGenesis ${ }^{\mathrm{TM}}$ Ltd for writing and editorial support for this publication. Dr M. Cottingham and Dr E. Southam of Oxford PharmaGenesis ${ }^{\text {TM }}$ Ltd provided writing assistance under the direction of the authors. Editorial assistance in editing, fact-checking, formatting, proofreading and submission was also provided by Oxford PharmaGenesis ${ }^{\mathrm{TM}}$ Ltd.

The following authors have received compensation for serving as consultants or speakers, or they, or the institutions they work for, have received research support or royalties from the companies or organizations indicated: T. Banaschewski (Hexal, Lilly, Medice, Novartis,
Otsuka, Oxford Outcomes, PCM Scientific, Shire and Vifor Pharma); M. Lecendreux (Shire, UCB and Vifor Pharma); M. Johnson (Janssen, Lilly, Otsuka, PCM Scientific, Shire, Vifor Pharma, and WM Lundgrens Research Fund); A. Zuddas (AstraZeneca, Bristol-Myers Squibb/Otsuka, Lilly, Lundbeck, Shire and Vifor Pharma); D. Coghill (Flynn, Janssen-Cilag, Lilly, Medice, Novartis, Otsuka, Oxford University Press, Pfizer, Schering-Plough, Shire, UCB and Vifor Pharma). B. Adeyi and L. A. Squires are employees of Shire, and may hold Shire stock and/or stock options. P. Hodgkins is a current employee of Vertex Pharmaceuticals and a former employee of Shire.

Open Access This article is distributed under the terms of the Creative Commons Attribution Noncommercial License which permits any noncommercial use, distribution, and reproduction in any medium, provided the original author(s) and the source are credited.

\section{References}

1. Pennick M. Absorption of lisdexamfetamine dimesylate and its enzymatic conversion to $d$-amphetamine. Neuropsychiatr Dis Treat. 2010;6:317-27.

2. Wigal SB, Kollins SH, Childress AC, Squires L. A 13-h laboratory school study of lisdexamfetamine dimesylate in schoolaged children with attention-deficit/hyperactivity disorder. Child Adolesc Psychiatry Ment Health. 2009;3:17.

3. Wigal T, Brams M, Gasior M, Gao J, Squires L, Giblin J. Randomized, double-blind, placebo-controlled, crossover study of the efficacy and safety of lisdexamfetamine dimesylate in adults with attention-deficit/hyperactivity disorder: novel findings using a simulated adult workplace environment design. Behav Brain Funct. 2010;6:34.

4. Biederman J, Krishnan S, Zhang Y, McGough JJ, Findling RL. Efficacy and tolerability of lisdexamfetamine dimesylate (NRP104) in children with attention-deficit/hyperactivity disorder: a phase III, multicenter, randomized, double-blind, forced-dose, parallel-group study. Clin Ther. 2007;29:450-63.

5. Findling RL, Childress AC, Cutler AJ, Gasior M, Hamdani M, Ferreira-Cornwell MC, et al. Efficacy and safety of lisdexamfetamine dimesylate in adolescents with attention-deficit/hyperactivity disorder. J Am Acad Child Adolesc Psychiatry. 2011;50:395-405.

6. Adler LA, Goodman DW, Kollins SH, Weisler RH, Krishnan S, Zhang Y, et al. Double-blind, placebo-controlled study of the efficacy and safety of lisdexamfetamine dimesylate in adults with attention-deficit/hyperactivity disorder. J Clin Psychiatry. 2008;69:1364-73.

7. Coghill D, Banaschewski T, Lecendreux M, Soutullo C, Johnson M, Zuddas A, et al. European, randomized, phase 3 study of lisdexamfetamine dimesylate in children and adolescents with attention-deficit/hyperactivity disorder. Eur Neuropsychopharmacol. 2013;23:1208-18.

8. Brams M, Weisler R, Findling RL, Gasior M, Hamdani M, Ferreira-Cornwell MC, et al. Maintenance of efficacy of lisdexamfetamine dimesylate in adults with attention-deficit/ hyperactivity disorder: randomized withdrawal design. J Clin Psychiatry. 2012;73:977-83.

9. Coghill DR, Banaschewski T, Lecendreux M, Johnson M, Zuddas A, Anderson CS, et al. Maintenance of efficacy of lisdexamfetamine dimesylate in children and adolescents with attentiondeficit/hyperactivity disorder: randomized-withdrawal study design. J Am Acad Child Adolesc Psychiatry. 2014;53: 647-57.e1. 
10. Goodman DW. Sustained treatment effect in attention-deficit/ hyperactivity disorder: focus on long-term placebo-controlled randomized maintenance withdrawal and open-label studies. Ther Clin Risk Manag. 2013;9:121-30.

11. Parens E, Johnston J. Facts, values, and attention-deficit hyperactivity disorder (ADHD): an update on the controversies. Child Adolesc Psychiatry Ment Health. 2009;3:1.

12. American Psychiatric Association. Neurodevelopmental disorders. Diagnostic and statistical manual of mental disorders. 5th ed. Arlington: American Psychiatric Publishing; 2013.

13. Banaschewski T, Soutullo C, Lecendreux M, Johnson M, Zuddas A, Hodgkins P, et al. Health-related quality of life and functional outcomes from a randomized, controlled study of lisdexamfetamine dimesylate in children and adolescents with attention deficit hyperactivity disorder. CNS Drugs. 2013;27:829-40.

14. Riley AW, Coghill D, Forrest CB, Lorenzo MJ, Ralston SJ, Spiel G. Validity of the health-related quality of life assessment in the ADORE study: Parent Report Form of the CHIP-Child Edition. Eur Child Adolesc Psychiatry. 2006;15(Suppl 1):I63-71.

15. Riley AW, Forrest CB, Starfield B, Rebok GW, Robertson JA, Green BF. The Parent Report Form of the CHIP-Child Edition: reliability and validity. Med Care. 2004;42:210-20.

16. Canadian Attention Deficit Hyperactivity Disorder Resource Alliance. Weiss Functional Impairment Rating Scale-Parent Report (WFIRS-P). 2000. http://www.caddra.ca/cms4/pdfs/ caddraGuidelines2011WFIRS_P.pdf. Accessed 18 Mar 2013.

17. Canadian Attention Deficit Hyperactivity Disorder Resource Alliance. Weiss Functional Impairment Rating Scale (WFIRS) instructions. 2000. http://www.caddra.ca/cms4/pdfs/caddra Guidelines2011WFIRSInstructions.pdf. Accessed 14 Oct 2013.

18. European Medicines Agency, Committee for Medicinal Products for Human Use. Guideline on the clinical investigation of medicinal products for the treatment of attention deficit hyperactivity disorder (ADHD). 2010. http://www.ema.europa.eu/docs/ en_GB/document_library/Scientific_guideline/2010/08/WC500095686.pdf. Accessed 22 Nov 2013.

19. Riley AW, Robertson JA, Forrest CB, Green BF, Rebok GW, Starfield B. Technical manual for the Child Health and Illness Profile-Child Edition (CHIP-CE) Parent and Child Report Forms (Version 1.0). Baltimore: Johns Hopkins University; 2001.

20. Riley A, Starfield B, Forrest CB, Green BF, Robertson J, Rajmil L. Child Health and Illness Profile: a comprehensive assessment of health and functioning for children and adolescents. 2007. http://www.childhealthprofile.org/index.asp?pageid=49. Accessed 20 Nov 2013.
21. Coghill D. Pragmatic measures in paediatric psychopharmacology: are we getting it right? Eur Neuropsychopharmacol. 2011;21:571-83.

22. Coghill D, Danckaerts M, Sonuga-Barke E, Sergeant J. Practitioner review: quality of life in child mental health-conceptual challenges and practical choices. J Child Psychol Psychiatry. 2009;50:544-61.

23. Danckaerts M, Sonuga-Barke EJ, Banaschewski T, Buitelaar J, Dopfner M, Hollis C, et al. The quality of life of children with attention deficit/hyperactivity disorder: a systematic review. Eur Child Adolesc Psychiatry. 2010;19:83-105.

24. Schacht A, Escobar R, Wagner T, Wehmeier PM. Psychometric properties of the quality of life scale Child Health and Illness Profile-Child Edition in a combined analysis of five atomoxetine trials. Atten Defic Hyperact Disord. 2011;3:335-49.

25. Estrada MD, Rajmil L, Serra-Sutton V, Tebe C, Alonso J, Herdman M, et al. Reliability and validity of the Spanish version of the Child Health and Illness Profile (CHIP) Child-Edition, Parent Report Form (CHIP-CE/PRF). Health Qual Life Outcomes. 2010;8:78.

26. Fuentes J, Danckaerts M, Cardo E, Puvanendran K, Berquin P, De Bruyckere K, et al. Long-term quality-of-life and functioning comparison of atomoxetine versus other standard treatment in pediatric attention-deficit/hyperactivity disorder. J Clin Psychopharmacol. 2013;33:766-74.

27. Maziade M, Rouleau N, Lee B, Rogers A, Davis L, Dickson R. Atomoxetine and neuropsychological function in children with attention-deficit/hyperactivity disorder: results of a pilot study. J Child Adolesc Psychopharmacol. 2009;19:709-18.

28. Stein MA, Waldman ID, Charney E, Aryal S, Sable C, Gruber R, et al. Dose effects and comparative effectiveness of extended release dexmethylphenidate and mixed amphetamine salts. J Child Adolesc Psychopharmacol. 2011;21:581-8.

29. Epstein JN, Weiss MD. Assessing treatment outcomes in attention-deficit/hyperactivity disorder: a narrative review. Prim Care Companion CNS Disord. 2012;14. doi:10.4088/PCC.11r01336.

30. Cohen J. A power primer. Psychol Bull. 1992;112:155-9.

31. Coghill D. The impact of medications on quality of life in attention-deficit hyperactivity disorder: a systematic review. CNS Drugs. 2010;24:843-66.

32. Michelson D, Buitelaar JK, Danckaerts M, Gillberg C, Spencer TJ, Zuddas A, et al. Relapse prevention in pediatric patients with ADHD treated with atomoxetine: a randomized, double-blind, placebo-controlled study. J Am Acad Child Adolesc Psychiatry. 2004;43:896-904. 